ARTICLE

\title{
Nucleation of amorphous shear bands at nanotwins in boron suboxide
}

Qi An${ }^{1}$, K. Madhav Reddy ${ }^{2,3, \star}$, Jin Qian ${ }^{1, \star}$, Kevin J. Hemker ${ }^{3}$, Ming-Wei Chen ${ }^{2} \&$ William A. Goddard III

The roles of grain boundaries and twin boundaries in mechanical properties are well understood for metals and alloys. However, for covalent solids, their roles in deformation response to applied stress are not established. Here we characterize the nanotwins in boron suboxide $\left(\mathrm{B}_{6} \mathrm{O}\right)$ with twin boundaries along the $\{0 \overline{1} 11\}$ planes using both scanning transmission electron microscopy and quantum mechanics. Then, we use quantum mechanics to determine the deformation mechanism for perfect and twinned $\mathrm{B}_{6} \mathrm{O}$ crystals for both pure shear and biaxial shear deformations. Quantum mechanics suggests that amorphous bands nucleate preferentially at the twin boundaries in $\mathrm{B}_{6} \mathrm{O}$ because the twinned structure has a lower maximum shear strength by $7.5 \%$ compared with perfect structure. These results, which are supported by experimental observations of the coordinated existence of nanotwins and amorphous shear bands in $\mathrm{B}_{6} \mathrm{O}$, provide a plausible atomistic explanation for the influence of nanotwins on the deformation behaviour of superhard ceramics.

\footnotetext{
${ }^{1}$ Department of Chemistry, Materials and Process Simulation Center, California Institute of Technology, 1200 East California Boulevard, Pasadena, California 91125, USA. ${ }^{2}$ WPI Advanced Institute for Materials Research, Tohoku University, Sendai 980-8577, Japan. ${ }^{3}$ Department of Mechanical Engineering, Johns Hopkins University, Baltimore, Maryland 21218, USA. * These authors contributed equally to this work. Correspondence and requests for materials should be addressed to W.A.G. (email: wag@wag.caltech.edu).
} 
G rain boundaries (GBs) and twin boundaries (TBs) are known to strengthen polycrystalline materials such as metals and ceramics, where GBs can impede dislocation movements, so that decreasing grains sizes can strengthen the material, the Hall-Petch relationship ${ }^{1-4}$. However, for grain sizes below some critical value, the dominant deformation mechanism becomes GBs sliding and migration so that the material strength decreases for further decreases in grain size $e^{5,6}$. These grain size effects have been studied extensively in metals but not in bulk nanocrystalline ceramics ${ }^{7,8}$. Dislocation migration so important in metals is absent in most ceramics, especially at low temperature, so that we can expect quite different roles of GBs and TBs in the deformation mechanisms of ceramics as compared with metals and alloys.

Nanotwins are expected to be much lower in energy than normal GBs and might strengthen materials more efficiently ${ }^{9}$. For metals, nanotwinned systems generally fail well below their theoretical strength limit because of dislocation nucleation and emission from boundaries or surface imperfections ${ }^{10}$. However, in ceramics, nanoscale twins have been observed to dramatically enhance the hardness of diamond and boron nitride ${ }^{11,12}$, inspiring our examination of the roles of nanotwinned structures on the deformation mechanism in ceramics and covalent solids.

As a prototype model system for examining how twin structures affect the mechanical response under deformation, we examined superhard boron suboxide $\left(\mathrm{B}_{6} \mathrm{O}\right)$ ceramic because $\mathrm{B}_{6} \mathrm{O}$ is capable of forming unusual profusely twinned crystals with icosahedral habits ${ }^{13}$. In addition, $\mathrm{B}_{6} \mathrm{O}$ is a promising hard material with a Vickers hardness of $45 \mathrm{GPa}$, which can be used in manufacture of cutting tools, abrasives and body armour ${ }^{14,15}$ (also quantum mechanics (QM) calculations predict a direct band gap of $\sim 3.0 \mathrm{eV}$, making it a candidate for a $p$-type transparent conducting oxide ${ }^{16}$ ). $\mathrm{B}_{6} \mathrm{O}$ has been observed experimentally to undergo brittle failure with formation of shear-induced amorphous bands ${ }^{17}$ similar to boron carbide $\left(\mathrm{B}_{4} \mathrm{C}\right)^{18,19}$. We previously reported $\mathrm{QM}$ and reactive force field molecular dynamics simulations showing that brittle failure in $\mathrm{B}_{4} \mathrm{C}$ arises from formation of higher density amorphous bands that result from fracturing the icosahedra because of reactions with the distorted $\mathrm{C}-\mathrm{B}-\mathrm{C}$ chains ${ }^{20,21}$. The intrinsic failure mechanism of $\mathrm{B}_{6} \mathrm{O}$ is not known. However, in $\mathrm{B}_{6} \mathrm{O}$, the chains connecting the icosahedra involve two weakly coupled $\mathrm{O}$ atoms so that we expect that the icosahedra might not fracture under pure shear conditions $^{22}$

In this study, we use the state-of-the-art spherical aberration (Cs) corrected scanning transmission electron microscopy (STEM) to examine the nanoscale twins found in hot pressed $\mathrm{B}_{6} \mathrm{O}$, showing that the TBs lie on the $\{0 \overline{1} 11\}$ family planes and the angle between $\{1 \overline{1} 0 \overline{1}\}$ and $\{0 \overline{1} 11\}$ planes is $72.0^{\circ} \pm 0.3^{\circ}$. Then we use QM at the Perdew-Burke-Ernzerhof density functional theory (DFT) level to predict the most favourable twins. We find them to be in the $\{0111\}$ family plane with angles of $72.0^{\circ}$, in excellent agreement with our experiments. Next, we used QM to perform shear deformation along the $\{0 \overline{1} 11\}$ twin plane, where we find that the maximum shear strength for shearing twinned $\mathrm{B}_{6} \mathrm{O}$ is $43.3 \mathrm{GPa}$, very close to the ideal shear strength of $43.5 \mathrm{GPa}$ for single crystal $\mathrm{B}_{6} \mathrm{O}$, and higher than values for perfect $\mathrm{B}_{4} \mathrm{C}$ $(39.0 \mathrm{GPa})^{20}$. Finally, we performed biaxial shear deformation to mimic indentation experiments for both perfect and twinned $\mathrm{B}_{6} \mathrm{O}$. The DFT simulations suggest that indentation conditions lead to deconstruction of the icosahedra, providing a plausible explanation for the amorphous band formation observed experimentally in $\mathrm{B}_{6} \mathrm{O}$. We predict that the maximum shear stress is $7.5 \%$ lower for twinned $\mathrm{B}_{6} \mathrm{O}$, and that amorphous bands might nucleate at the nanoscale twins, which are supported by experimental observations of the coordinated existence of nanoscale twins and amorphous shear bands. These results indicate that $\mathrm{TBs}$ play an essential role in the deformation mechanism and intrinsic brittle failure of $\mathrm{B}_{6} \mathrm{O}$.

\section{Results}

Perfect and nanotwinned $\mathbf{B}_{6} \mathbf{O}$ structures. The crystalline structure of $\mathrm{B}_{6} \mathrm{O}$ consists of one $\mathrm{B}_{12}$ icosahedron per rhombohedral unit cell (space group $R \overline{3} m$ ), as shown in Fig. 1a, which is similar to $\mathrm{B}_{4} \mathrm{C}, \mathrm{B}_{12} \mathrm{P}_{2}$ and $\alpha$-boron ${ }^{22}$. The $\mathrm{B}_{12}$ icosahedra are connected via pseudo chains consisting of a pair of oxygen $(\mathrm{O}-\mathrm{O})$ atoms along the $\langle 0001\rangle$ direction. Here each $\mathrm{O}$ makes $\mathrm{B}-\mathrm{O}$ sigma bonds to three icosahedra, but the $\mathrm{O}-\mathrm{O}$ distance of $3.07 \AA$ is far too long for a bond. Thus, each $\mathrm{O}$ is formally $\mathrm{O}^{+}$, transferring one electron to a $B_{12}$ icosahedron, leading to 26 skeleton electrons within the icosahedron to satisfy Wade's rule ${ }^{23}$. Each icosahedron also makes six direct B-B sigma bonds to adjacent icosahedra. Annular bright-field STEM (ABF-STEM; Fig. 1b) shows the atomic image of perfect structure of $\mathrm{B}_{6} \mathrm{O}$ along the $\langle 10 \overline{1} 1\rangle$ projection. Comparing the experimental and computer simulated ABF-STEM images (inset bottom right) reveals 12-atom boron icosahedra $\left(B_{12}\right)$ in the form of atomic rings with two oxygen atoms distinguishably appearing as a dark-dot contrast linking the icosahedra. This can be recognized as oxygen atomic columns positioned to be consistent with the structural model of $\mathrm{B}_{6} \mathrm{O}$ in Fig. 1a. Figure 1c shows representative nanoscale twins observed with ABF-STEM showing that the twin bands range in width from sub-nanometre to a few nanometres within the $\mathrm{B}_{6} \mathrm{O}$ grains (white lines). The TBs lie on the $\{0 \overline{1} 11\}$ family planes, which agree very well with previous studies on twinned $\mathrm{B}_{6} \mathrm{O}$ and $\mathrm{B}_{4} \mathrm{C}$
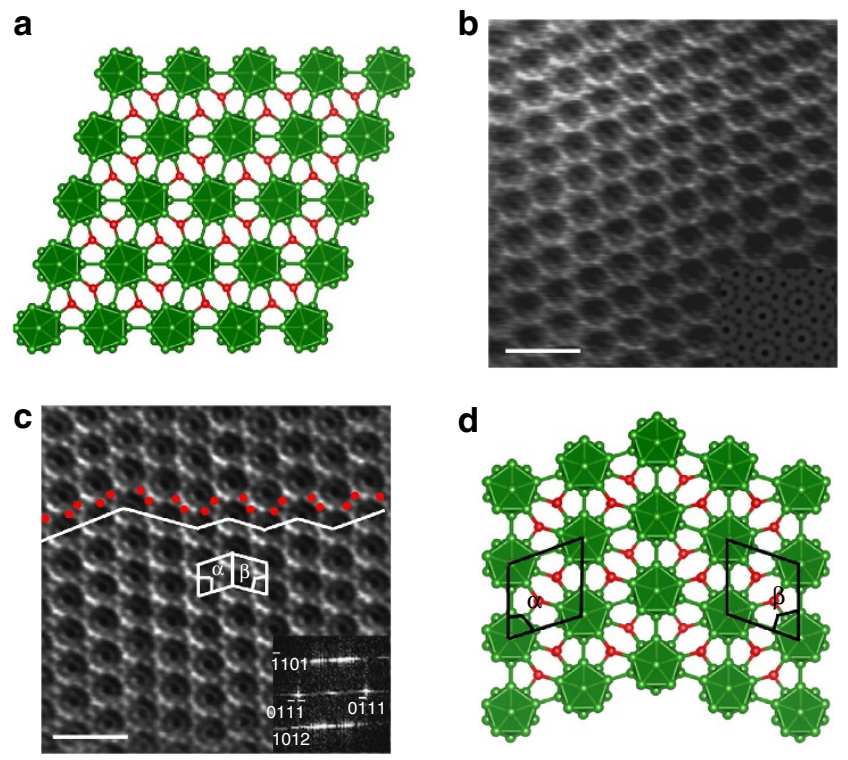

Figure 1 | The perfect and twinned $B_{6} \mathbf{O}$ structures from QM and STEM images. (a) The $\mathrm{B}_{6} \mathrm{O}$ rhombohedral structure, icosahedral $\mathrm{B}_{12}$ clusters (green colour) and linked oxygen atoms (red colour). (b) The annular bright-field STEM (ABF-STEM) image showing the $\mathrm{B}_{12}$ icosahedra as atomic rings linked between two oxygen atoms along $\langle 0001\rangle$ direction. The simulated ABF-STEM image of $\mathrm{B}_{6} \mathrm{O}$ is inserted at the bottom side. (c) A representative ABF-STEM image showing highly dense nanoscale twin bands within a grain and along the $\langle 10 \overline{1} 1\rangle$ zone axis. Images have been smoothed and Fourier filtered to enhance the contrast of nano-twinned $\mathrm{B}_{6} \mathrm{O}$ atomic structure (see Supplementary Fig. 9). (b,c) Scale bars, $1 \mathrm{~nm}$. (d) The twinned $\mathrm{B}_{6} \mathrm{O}$ structure from $\mathrm{QM}$. The measured angles on both sides of the TBs are $\alpha=72.0^{\circ}$ and $\beta=72.0^{\circ}$, which are consistent agree well with the experimental values of $72.0^{\circ} \pm 0.3^{\circ}$ as shown in FFT pattern (inset $\mathbf{c}$ ). 
(refs 13,24-26). In addition, the ABF-STEM image and inset fast Fourier transform (FFT) pattern demonstrate clearly that the angles between $\{1 \overline{1} 0 \overline{1}\}$ and $\{0 \overline{1} 11\}$ planes are $72^{\circ}$ on both sides of the TBs, indicating symmetric twins in $\mathrm{B}_{6} \mathrm{O}$.

To interpret the experimentally observed twinned structure in $\mathrm{B}_{6} \mathrm{O}$, we used $\mathrm{QM}$ to determine the atomic geometry of the twin, leading to the results in Fig. 1d. Our QM unit cell considered total four layers of icosahedra perpendicular to the $\{0 \overline{1} 11\}$ twin plane, leading to two TB planes per unit cell. We also constructed an eight-layer twinned structure to examine the multiple layer effects. These QM twin models can be compared with experimental structures having only several layers of icosahedra, as shown in Fig. 1c. The QM predicts angles on both sides of the TBs of $\alpha=72.0^{\circ}$ and $\beta=72.0^{\circ}$ in excellent agreement with our experimental value of $72.0^{\circ} \pm 0.3^{\circ}$. Moreover, the $\mathrm{QM}$ predicts an interfacial energy for the TB of only $-1.69 \mathrm{~mJ} \mathrm{~m}^{-2}$, which is much smaller than the stacking faults energy in most metals and ceramics $\left(>20 \mathrm{~mJ} \mathrm{~m}^{-2}\right)^{27,28}$. This low interfacial energy may explain the unusual highly twinned crystals that we found in the experimentally synthesized $\mathrm{B}_{6} \mathrm{O}$.

Deformation mechanism of nanotwinned $\mathrm{B}_{\mathbf{6}} \mathrm{O}$. The existence of large numbers of nanoscale twins in $\mathrm{B}_{6} \mathrm{O}$ might be expected to dramatically change the mechanical properties, especially the strength and failure mechanism. To investigate these effects, we used QM to describe the effect of shearing the twinned supercell $\mathrm{B}_{6} \mathrm{O}$ parallel the $\{0 \overline{1} 11\}$ twin plane. The computed stress-strain relationship for the twinned structure is shown in Fig. 2a and is compared with that of the perfect $\mathrm{B}_{6} \mathrm{O}$ (ref. 22). The slope of the stress-strain curve for the twinned structure is $7.5 \%$ lower than that of perfect $\mathrm{B}_{6} \mathrm{O}$, confirming the softening effect of the TBs. The maximum shear stress is $43.3 \mathrm{GPa}$ for the twinned structure, very close to our calculated ideal shear stress of $43.5 \mathrm{GPa}$ (ref. 22). This indicates that the nanotwinned structure could have the theoretical strength. It is well known that $\mathrm{B}_{6} \mathrm{O}$ is anisotropic and the lower strength for twinned structure might arise from the anisotropic effects. Thus, we also sheared the perfect crystal along the $\langle\overline{1} 01 \overline{1}\rangle$ (opposite direction of $\langle 10 \overline{1} 1\rangle$ ) and obtained a higher maximum shear stress of $51.0 \mathrm{GPa}$ because of the directional of covalent bonding in $\mathrm{B}_{6} \mathrm{O}$. This indicates that the twinned structure has intrinsically lower strength than perfect $\mathrm{B}_{6} \mathrm{O}$.

As the twinned structure is sheared, we observed that the middle layers (Fig. 2b) start to shear along the $\langle 10 \overline{1} 1\rangle$ easy direction. However, the other layers shear along the opposite hard shear direction of $\langle\overline{1} 01 \overline{1}\rangle$. As the shear stress increases to its maximum at 0.276 strain, a B-B bond (black oval in Fig. 2b) between icosahedra increases from $1.72 \AA$ in the intact structure (Fig. 2b) to $2.31 \AA$ (Fig. 2c). To analyse the bonding, we use the electron localization function ${ }^{29}$ shown in Supplementary Fig. 1a,b, which indicates that the electron pair in the B-B covalent bond is not broken until the shear strain increases to 0.345 (Fig. 2d). After breaking the B-B bond, the icosahedra in the middle layer (indicted by the black arrowhead in Supplementary Fig. 1b) have only ten extra-cage bonds so that they do not need extra electrons from nearby $\mathrm{O}$ atoms to satisfy Wade's rule. The black oval in Fig. $2 \mathrm{~d}$ shows that the $\mathrm{B}-\mathrm{O}$ bond distance increases from 1.51 to $1.64 \AA$, indicating that some covalent $\mathrm{B}-\mathrm{O}$ bonding changes to donor-acceptor character. In the starting structure, the $\mathrm{O}$ is effectively $\mathrm{O}^{+}$, which like $\mathrm{N}$ can make three covalent bonds, leaving a lone pair pointing at the other $\mathrm{O}$; but for the strained system, the $\mathrm{O}$ is neutral so that it can make two covalent bonds and one donor-acceptor (Lewis baseLewis acid) bond, which get averaged over the three $\mathrm{B}-\mathrm{O}$ linkages. The icosahedra near the middle layer (indicated by the red arrowhead in Supplementary Fig. 1b) now also have ten extra- a

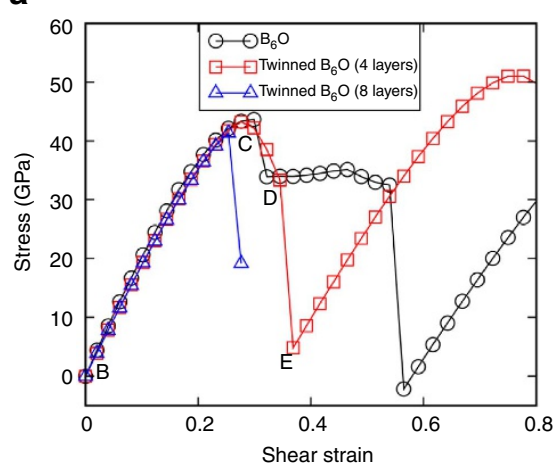

b
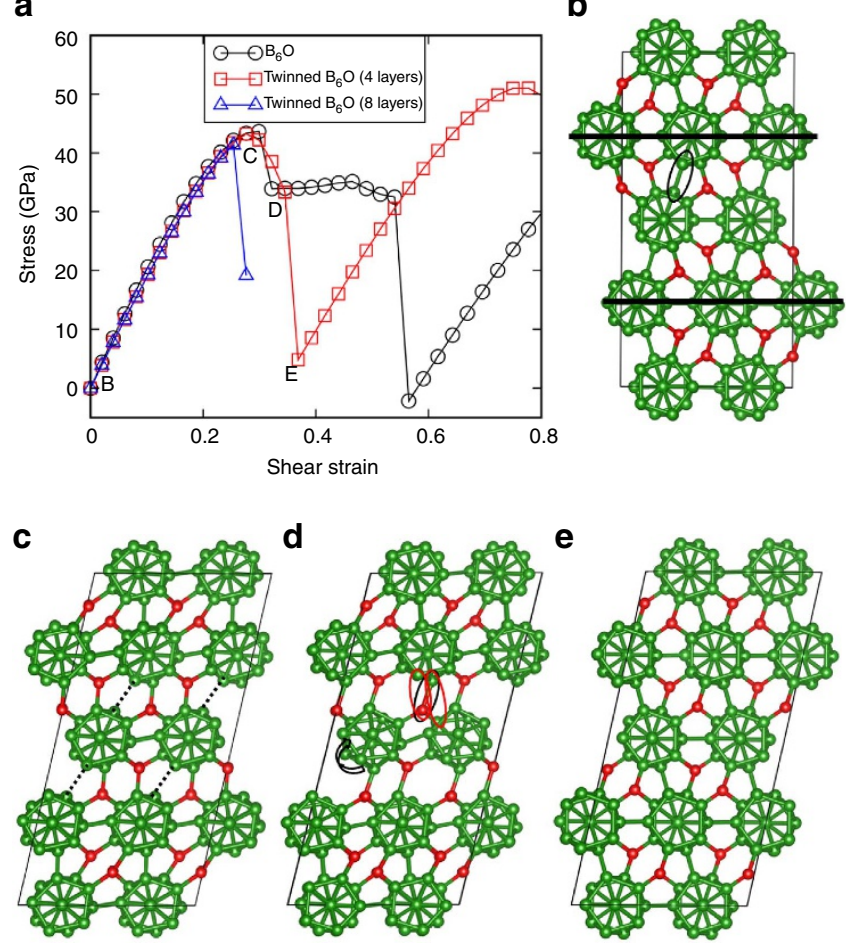

Figure 2 | Pure shear deformation along the $\{0111\}$ twin plane for the twinned structure compared with perfect $\mathbf{B}_{\mathbf{6}} \mathbf{O}$. (a) Stress-strain relationship. (b) Initial twin structure at 0.0 strain. The twin boundary is represented by solid black line. The B-B bond within the oval breaks in the following shear steps. (c) Structure at 0.276 strain, which corresponds to the maximum stress. The stretched B-B bonds are represented by dashed lines. (d) Structure at 0.345 strain, before the structure transformation. The donor-acceptor $\mathrm{B}-\mathrm{O}$ bond is within the black oval and the forming $\mathrm{B}-\mathrm{O}$ and B-B bond are within the red ovals. (e) Structure recovery to perfect crystal at 0.369 strain.

cage bonds because one $\mathrm{B}-\mathrm{O}$ bond becomes a donor-acceptor bond with only one broken B-B bond between icosahedra. This leads to a slight rotation of the icosahedral cluster in the middle layer, as shown in Fig. 2d. Finally, as the shear strain increases continuously to 0.369 , the donor-acceptor $\mathrm{B}-\mathrm{O}$ bond breaks, a new B-B covalent bond forms between the cages, and a $\mathrm{B}-\mathrm{O}$ covalent cage chain bond forms (red ovals in Fig. 2d). The result is to reconstruct the perfect crystal structure, as shown in Fig. 2e.

It is of interest to determine whether structure recovery happens at finite temperature for fast strain rates. To address this issue, we performed ab initio molecular dynamics (AIMD) simulations assuming constant volume shear deformation along the same slip system at room temperature using the strain rate of $1.0 \times 10^{10} \mathrm{~s}^{-1}$. Here we fixed the volume in the MD simulations to mimic the high strain rate deformation. The stressstrain relationship and the structural changes displayed in Supplementary Fig. 2 show that there is structure recovery. Thus, we find that the original twinned structure recovers back to twinned structure, which is different from what we find for static shear deformations where the twinned structure transforms to a perfect crystal structure. Thus, we conclude that the twinned structure will recover to itself during high strain rate shear deformations at room temperature.

To examine how the multiple layers between TBs might affect the deformation mechanism described above, we constructed the eight-layer twinned structure as shown in Supplementary Fig. 3a and applied a pure shear deformation to it. The stress-strain 
relationship in Fig. 2a shows that the maximum shear stress decreases from 43.3 to $41.4 \mathrm{GPa}$ as the number of layers between TBs increases from 2 to 4, indicating a weakening effect because of the multiple layer structure. The structural changes for this eight-layer twinned structure under pure shear deformation are displayed in Supplementary Fig. 3b,c. As the shear strain increases to 0.254 , which corresponds to the maximum shear stress, the B-B bonds between icosahedra (within the region of the easy shear direction) break, as shown in Supplementary Fig. $3 \mathrm{~b}$. As the shear strain increases to 0.276 , the middle two layers within the easy shear region recover to the crystal structure with the direction along the hard shear direction (the other twinned region). This leads to one-layer twin structure (or stacking fault) within a new eight-layer structure, as shown in Supplementary Fig. 3c. No icosahedra break in this shear process, which is similar to the four-layer twinned and perfect $\mathrm{B}_{6} \mathrm{O}$ structures ${ }^{22}$.

Although the twinned structure has intrinsically lower strength than perfect $\mathrm{B}_{6} \mathrm{O}$, the eight-layer twinned structure is even weaker than four-layer twinned structure. In the sheared deformation of the twinned structure, the half layer is sheared along the $\langle 10 \overline{1} 1\rangle$ easy direction, whereas the other half layer is sheared along the hard shear direction of $\langle\overline{1} 01 \overline{1}\rangle$. In the four-layer twinned structure, both the two easy sheared layers are directly bonded to the two hard sheared layers, which make the TBs harder to slip than eight-layer twinned structure where only the boundary layers are bonded to hard sheared layers.

Our simulations suggest a weakening effect from TBs compared with the ideal strength of the perfect crystal. The ideal strength of single crystals is relevant only for incipient plasticity. Previous experimental studies infer the hardening effects of twins in diamond and $c-\mathrm{BN}^{11,12}$ based on the hardness measurements under conditions of fully developed plasticity. No ideal crystal exists in these conditions. Further study is required to understand the twin hardening mechanism for diamond $/ c-B N$ under experimental conditions.

The shear simulations on both twinned $\mathrm{B}_{6} \mathrm{O}$ and perfect $\mathrm{B}_{6} \mathrm{O}$ lead to structural recovery without breaking the icosahedra. This suggests a critical design element for designing ductile hard materials of boron suboxide and related materials. The key is to form donor-acceptor bonds between the icosahedra and chain structures that can break the cage-chain bonds to relax the high stress without breaking the icosahedra. This prevents amorphous band formation, leading to improved ductility of superhard ceramics.

Nano-indentation on nanotwinned $\mathbf{B}_{6} \mathbf{O}$. To characterize the shear deformation of $\mathrm{B}_{6} \mathrm{O}$ experimentally, we carried out nano-indentation experiments on highly dense nanotwinned bulk specimens and prepared a cross-sectioned thin foil of the area beneath the indent using FIB milling. Conventional transmission electron microscopy (TEM) and STEM observations were used to examine the underlying mechanism.

Figure 3 shows the TEM and STEM images of the indentation region of $\mathrm{B}_{6} \mathrm{O}$. The low-magnification TEM image (Fig. 3a) shows several micro-crack regions and amorphous shear bands, suggesting that severe plastic deformation and damage take place underneath the indenter. The higher magnification TEM images (Fig. 3b and Supplementary Fig. 4) indicate that the nanoscale amorphous band was formed in close proximity to and intersects numerous nanoscale twins. The amorphous band is $200-300 \mathrm{~nm}$ long with a 1- to 3 -nm width. The nanoscale twin bands and the amorphous shear band are highlighted with black and white arrowheads in Fig. 3b-d). The atomic structure of the amorphous $\mathrm{B}_{6} \mathrm{O}$ band, viewed along the $\langle 10 \overline{1} 1\rangle$ zone axis, is shown in Fig. $3 \mathrm{c}$;
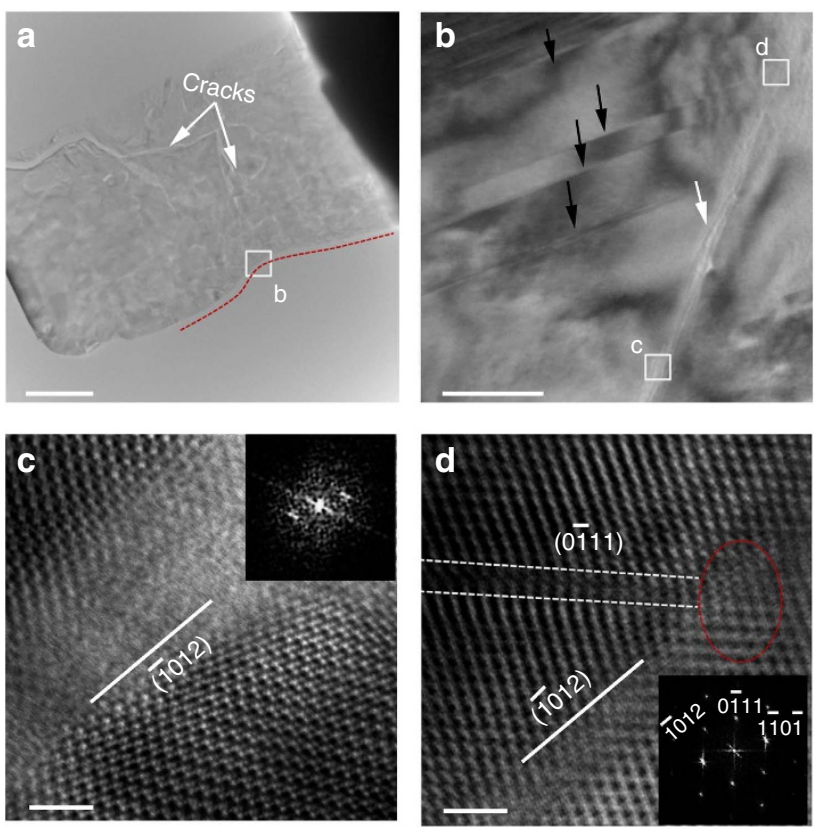

Figure 3 | TEM images of the cross-sectioned indentation region.

(a) Low-magnification TEM beneath the indentation region. Scale bar, $2 \mu \mathrm{m}$. (b) Magnified TEM image beneath the indented region showing a shear band (white arrowhead) in the highly dense twin grain (black arrowhead) from the $\langle 10 \overline{1} 1\rangle$ projection. Scale bar, $50 \mathrm{~nm}$. (c) ABF-STEM taken from the shear band reveals the loss of periodicity in a $\mathrm{B}_{6} \mathrm{O}$ grain. Inset FFT pattern demonstrate the amorphous nature of the shear band. The crystalline of $\mathrm{B}_{6} \mathrm{O}$ can be found on both sides of amorphous band. (d) ABF-STEM image at the tip of amorphous shear band (red circle) shows dissociated icosahedral clusters initiated from a twin bands. The white box is to help distinguish twin structure. (c,d) Scale bar, $2 \mathrm{~nm}$.

crystalline $\mathrm{B}_{12}$ icosahedra are visible on both sides, but a loss of periodicity is evident within the $\sim 3 \mathrm{~nm}$ band. A high-resolution STEM image of this amorphous band is shown in Fig. $3 \mathrm{~d}$ and indicates that amorphous $\mathrm{B}_{6} \mathrm{O}$ band formation occurs along the $\{\overline{1} 012\}$ plane and intersects at their close proximity of observed nanoscale twin plane. In Fig. $3 c$,d, white dots correspond to the central positions of the $\mathrm{B}_{12}$ icosahedral clusters as suggested by image simulation ${ }^{30}$. In Fig. $3 d$, the amorphous band is less than $1 \mathrm{~nm}$ in width, as compared with $3 \mathrm{~nm}$ in Fig. 3c. The measured angle between the amorphous band plane and the twin plane is about $\sim 54^{\circ}$.

Our previous study ${ }^{21}$ showed that the amorphous band has higher density than the crystalline region because of deconstruction of the icosahedral clusters. This leads to the severe lattice distortion in the local amorphous region that likely causes the different angles between $\{0 \overline{1} 11\}$ planes compared with the intact crystal. The FFT patterns in Fig. 3d from localized region around amorphous band have angular mismatch $\sim 3^{\circ}$ of crystals on either side of the amorphous band as compared with predicted angle. However, selected area diffraction patterns taken from large areas (few hundred nanometres) on either side of amorphous band as shown in Supplementary Fig. 4 are consistent with the theoretical angle of $72.0^{\circ}$ of $\{0 \overline{1} 11\}$ planes. These observations confirm the severe deformation of crystalline region near the amorphous band in $\mathrm{B}_{6} \mathrm{O}$.

Deformation mechanisms under indentation. To understand the amorphous shear band formation mechanism observed in the 

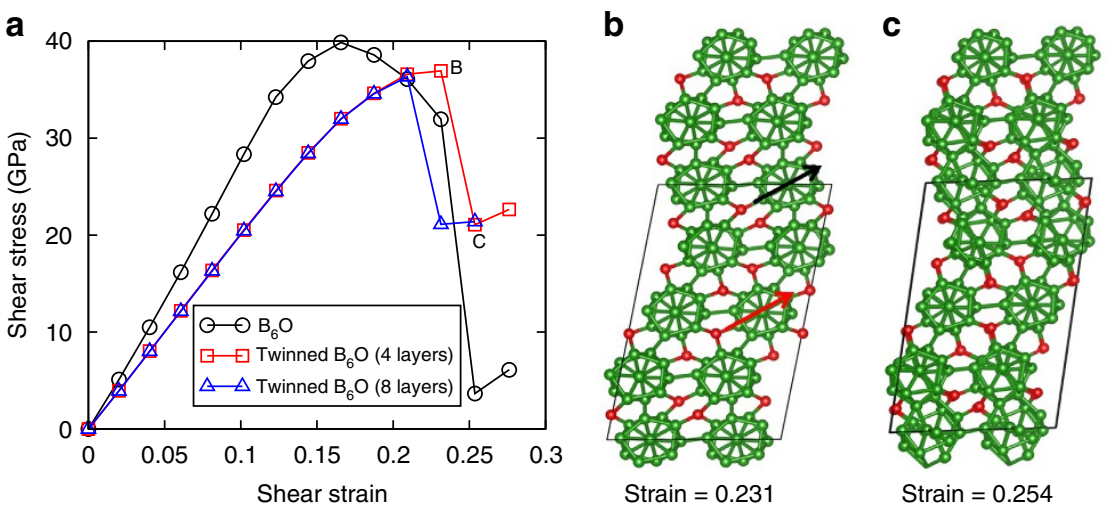

Figure 4 | Biaxial shear deformation of twinned $\mathrm{B}_{6} \mathrm{O}$ shearing along the $\{0111\}$ twin plane to mimic an indentation experiment, which is compared with the perfect $\mathbf{B}_{\mathbf{6}} \mathbf{O}$. (a) Stress-strain relationship. (b) Structure at 0.231 strain before failure. The long axis of the most distorted and the least icosahedra are represented by black and red arrowhead, respectively. (c) Structure at 0.254 strain after failure.

indentation experiments, we used DFT to perform biaxial shear deformations. The stress-strain relationships under biaxial shear deformation for both twinned and perfect $\mathrm{B}_{6} \mathrm{O}$ are shown in Fig. 4a. In contrast to pure shear deformation, we calculate that the slope of the stress-strain relationship for twinned structure is $23.4 \%$ smaller than the perfect $\mathrm{B}_{6} \mathrm{O}$, indicating that $\mathrm{TBs}$ under indentation induce a pronounced softening effect. The maximum shear stress for twinned $\mathrm{B}_{6} \mathrm{O}$ is $36.9 \mathrm{GPa}$, which is $8 \%$ lower than for the perfect $\mathrm{B}_{6} \mathrm{O}(39.9 \mathrm{GPa})$. As we discussed in the pure shear deformation, the twinned structure has intrinsically lower strength than perfect $\mathrm{B}_{6} \mathrm{O}$ because of interfacial energy from twins. This suggests that amorphous bands might nucleate at the TBs under indentation conditions. Other defects such as vacancies and GBs might also be important in the experiments on amorphous band formation.

Under biaxial shear conditions, we find that compression perpendicular to the TBs plays an essential role in the disintegration of icosahedra. As the shear stress reaches its maximum value of $36.9 \mathrm{GPa}$ at 0.231 strain, no bonds break, as shown in Fig. 4b. Instead, the icosahedra deform to an ellipsoid shape that accommodates the applied biaxial stress. The most deformed icosahedra are in the layer that shears along the hard direction. This can be characterized by the $\mathrm{B} \cdots \mathrm{B}$ distance (opposite to each other in the cage, indicated by black arrowhead in Fig. $4 \mathrm{~b}$ ), which increases from 3.39 to $3.66 \mathrm{~A}$. In contrast, this distance is $3.60 \AA$ for the least distorted icosahedra located in the layer (red arrowhead in Fig. 4b) that is shearing along the easy direction. This deconstructs the icosahedra layers along the hard shear directions as the strain increases to 0.254 , as shown in Fig. 4c.

To examine how the multiple layers between TBs affect the biaxial deformation mechanism, we applied biaxial shear deformation to the eight-layer twinned structure. The maximum shear stress for the eight-layer twinned $\mathrm{B}_{6} \mathrm{O}$ is $36.3 \mathrm{GPa}$, which is very slightly lower than the four-layer twinned structure of $36.9 \mathrm{GPa}$, as shown in Fig. 4a. The biaxial deformation mechanism for eight-layer twinned structure is similar to fourlayer twinned structure where the icosahedra within the hard shear direction are disintegrated under the effects of both compression and shear, as shown in Supplementary Fig. 5.

The structural changes of perfect $\mathrm{B}_{6} \mathrm{O}$ under indentation conditions are shown in Supplementary Fig. 6a-d. As the shear stress reaches its maximum of $39.9 \mathrm{GPa}$ at 0.166 strain, the icosahedra deform without breaking any bonds (Supplementary Fig. 6a). The B-B bond between icosahedra along the $\langle 10 \overline{1} 1\rangle$ direction stretches from 1.70 to $1.99 \AA$. As the shear strain continuously increases farther to 0.231 , the shear stress decreases

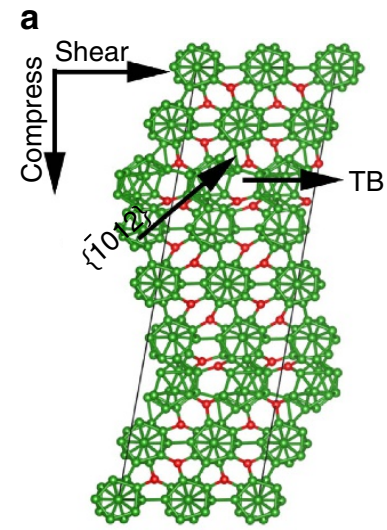

b

Figure 5 | Deformation mechanism for forming an amorphous shear band at realistic experimental conditions. (a) The biaxial shear deformation of four-layer nanotwins leads to deconstruction of icosahedra along the TB plane, corresponding to the red circle in Fig. 3d. (b) The biaxial shear deformation of perfect crystal leads to deconstruction of icosahedra along $\{1012\}$ plane, which aligns the amorphous band propagation in Fig. 3c,d.

to $31.9 \mathrm{GPa}$. The icosahedra deform to an ellipsoidal shape (Supplementary Fig. 6b), where the $\mathrm{B} \cdots \mathrm{B}$ distance for the long axis along the $\langle 1 \overline{1} 02\rangle$ direction (opposite to each other along the $\langle 1 \overline{1} 02\rangle)$ increases to $3.89 \AA$. This leads to deconstruction of icosahedra as the shear strain increases to 0.254 , as shown in Supplementary Fig. 6c. Not all icosahedra in the simulation cell break. Instead, the icosahedra deconstruct along the alternative layers of $\{\overline{1} 102\}$ planes, as shown in the rotated structure of Supplementary Fig. 6d.

\section{Discussion}

Combining the experimental TEM observations with the QMderived deformation mechanism under biaxial deformation, we propose a deformation mechanism in which the amorphous band initiates at the nanoscale twins and then it propagates along the $\{\overline{1} 012\}$ family plane, as shown in Figs 3 and 5 . The process can be described as follows. First, two layers of icosahedra along the TBs disintegrate under the indentation conditions, as shown in Fig. 5a. This appears to trigger the formation of amorphous bands that are seen in the experimental images (see, for example, Fig. 3d). Then the deconstructed icosahedra in the TB plane make it easier to form an amorphous band in the perfect $\mathrm{B}_{6} \mathrm{O}$, as shown in Fig. 5b. The orientation of the amorphous band extends along the $\{1012\}$ plane, which is not aligned along the TB plane but is 
consistent with the simulations of biaxial deformation of $\mathrm{B}_{6} \mathrm{O}$ described above.

The transition of the amorphous band from TB plane to $\{\overline{1012}\}$ plane may relate to the complex stress conditions in nanoindentation experiments. Based on the above QM study, the amorphous band formed along the TB plane is under the biaxial stress conditions where the shear stress is along the TB plane and the compressive stress is perpendicular to the TB plane (Fig. 5a). As the amorphous band changes and propagates along the $\{1012\}$ plane in the perfect $\mathrm{B}_{6} \mathrm{O}$, the shear stress is still along the TB plane, whereas the compressive stress is along the $\langle 1101\rangle$ direction, as shown in Fig. 5b.

Our early study ${ }^{17}$ showed that nucleation of amorphous bands is possible in the perfect crystal, but our current experimental observation suggests that they are easier to nucleation in the vicinity of twins. This finding is in good agreement with the QM calculations that find the twinned structure to have a lower maximum shear strength by $7.5 \%$ compared with perfect crystal structure.

Figure $3 \mathrm{~b}$ shows that the amorphous bands propagate across the twin bands without obvious turning. Our QM simulations on the biaxial shear deformation of the twinned structure find deconstruction of the icosahedra only in the TBs and the hard shear region, which is consistent with the TEM images shown in Supplementary Fig. 7 (white dots correspond to the central positions of the $\mathrm{B}_{12}$ icosahedral clusters). This suggests some discontinuity might exist in the twinned structure. However, the twin bands are too narrow to observe the discontinuity in our experimental images. Our current paper focuses on the nanoscale twins in $\mathrm{B}_{6} \mathrm{O}$ and their effects to the initiation of the amorphous band formation. It will be interesting to later examine the amorphous band propagation after initiation.

The QM calculations find that the amorphous band in $\mathrm{B}_{6} \mathrm{O}$ is along $\{\overline{1} 012\}$ that is different from $\mathrm{B}_{4} \mathrm{C}$, which is along $\{01 \overline{1} \overline{1}\}^{18}$. To compare the mechanism for these two systems, we performed biaxial shear simulations on $\mathrm{B}_{4} \mathrm{C}$ perfect crystal leading to the structural changes shown in Supplementary Fig. 8. For $\mathrm{B}_{4} \mathrm{C}$, the deconstruction of icosahedra is still along $\{01 \overline{1} 1\}$ family plane because the failure of $\mathrm{B}_{4} \mathrm{C}$ arises from the interaction of the $\mathrm{C}-\mathrm{B}-$ $\mathrm{C}$ chain with the $\mathrm{B}_{11} \mathrm{C}$ icosahedral cluster. In contrast for $\mathrm{B}_{6} \mathrm{O}$, the $\mathrm{C}-\mathrm{B}-\mathrm{C}$ chain is replaced by two individual $\mathrm{O}$ atoms, which significantly changes the deformation and failure mechanism.

In this work, we identified the atomistic structure of nanoscale twins in $\mathrm{B}_{6} \mathrm{O}$ using a combination of TEM experiments and $\mathrm{QM}$ simulations. Then, we used QM to determine the shear deformation mechanism of twinned structures, finding that the twin can transform back to the crystalline structure. This is similar to the structural recovery in perfect $\mathrm{B}_{6} \mathrm{O}$. These results indicate that pure shear deformation need not form an amorphous shear band for either perfect or twinned $\mathrm{B}_{6} \mathrm{O}$.

Then, we investigated the deformation mechanism for twinned and perfect $\mathrm{B}_{6} \mathrm{O}$ under indentation conditions with $\mathrm{QM}$ biaxial shear simulations. Here we found that for the perfect crystal the layer deconstructs in alternate layers of $\{\overline{1} 012\}$ family planes, with a maximum shear stress of $39.9 \mathrm{GPa}$. In contrast, for the twinned system, the amorphous band forms more easily when shear is along the twin plane, with a maximum shear stress of 36.9 GPa. Thus, it is plausible that amorphous shear bands nucleate at the nanoscale twins, which is supported by indentation experimental observation of the co-existence of nanoscale twins and amorphous shear bands.

Summarizing, we demonstrate that combining STEM nanomechanical testing with QM modelling can provide a deeper understanding of the fundamental deformation mechanisms in ceramics, which may enable the design of ductile superhard ceramics.

\section{Methods}

Computational details. All simulations were performed used the Vienna Ab-initio Simulation Package (VASP) periodic code with plane wave basis sets ${ }^{31-33}$. We used an energy cutoff of $600 \mathrm{eV}$ in all the simulations as it gives excellent convergence on energy, force, stress and geometries. We used the projector-augmented wave pseudopotentials for the Perdew-Burke-Ernzerhof exchange-correlation functional ${ }^{34}$. The energy error for terminating electronic self-consistent field and the force criterion for the geometry optimization were set equal to $10^{-6} \mathrm{eV}$ and $10^{-3} \mathrm{eV} \AA^{-1}$, respectively. The Monkhorst-Pack grid $(8 \times 8 \times 8)$ in the $k$-space was used in the unit cell geometry optimization. Later, a smaller grid $(2 \times 2 \times 2)$ and larger supercell $(2 \times 2 \times 2)$ were used in the biaxial shear deformations. The Monkhorst-Pack grid $(2 \times 4 \times 1)$ was used for the twinned $\mathrm{B}_{6} \mathrm{O}$ simulations.

To examine shear deformation, we imposed the strain for a particular shear plane while allowing full structure relaxation for the other five strain components ${ }^{35}$.

We mimic the indentation process by applying biaxial shear deformation. Here we considered the normal compressive pressure beneath the indenter and constrained the ratios of normal stress $\left(\boldsymbol{\sigma}_{\mathbf{z z}}\right)$ to shear stress $\left(\boldsymbol{\sigma}_{\mathbf{x z}}\right)$ as $\boldsymbol{\sigma}_{\mathbf{z z}}=\sigma_{\mathbf{z x}} \tan \Phi$ where $\Phi=68^{\circ}$ for a Vickers indentor ${ }^{36}$. The residual stresses after relaxing the other strain components in both shear and biaxial shear deformations were less than $0.5 \mathrm{GPa}$.

Material fabrication and nanoindentation. The fully dense $\mathrm{B}_{6} \mathrm{O}$ was synthesized using hot-pressing techniques at $2,173 \mathrm{~K}, 60 \mathrm{MPa}$ for $1 \mathrm{~h}$. The synthesis procedure is described in detail elsewhere ${ }^{37}$. We performed a series of indentations on the smoothly polished $\mathrm{B}_{6} \mathrm{O}$ surface at a constant loading force $(2.0 \mathrm{~N})$ and loading rate $\left(14.0 \mathrm{mN} \mathrm{s}^{-1}\right.$ ) using a dynamic ultra-microhardness tester (Shimadzu W201S) equipped with a Berkovich diamond indenter. The cross-sectional TEM specimens of the indented $\mathrm{B}_{6} \mathrm{O}$ were prepared by a lift-out technique ${ }^{38}$ using a FIB system (JEOL, JIB-4600F). Before the TEM observations, the TEM sample was further gently milled below $500 \mathrm{eV}$ by a Fashone 1,040 Nanomill system to remove the damage layer on the sample surface without altering the specimen chemistry and structure.

Transmission electron microscopy. We characterized the microstructure of assynthesized $\mathrm{B}_{6} \mathrm{O}$ using a JEM-2100 F transmission electron microscope (JEOL Ltd.) equipped with double spherical aberration correctors (CEOS $\mathrm{GmbH}$ ) for the image- and probe-forming lens systems. The annular bright-field STEM (ABFSTEM) images acquired a detector angle of $\sim 12-23 \mathrm{mrad}$ and point-to-point resolution $0.1 \mathrm{~nm}$. ABF-STEM image simulations were performed using a software package of Win HREM (HREM Research Inc).

\section{References}

1. Hall, E. O. The deformation and ageing of mild steel: III discussion of results. Proc. Phys. Soc. Lond. Ser. B 64, 747-753 (1951).

2. Petch, N. J. The cleavage strength of polycrystals. J. Iron Steel Inst 174, 25-28 (1953).

3. Meyers, M. A., Mishra, A. \& Benson, D. J. Mechanical properties of nanocrystalline materials. Prog. Mater. Sci. 51, 427-556 (2006).

4. Wollmershauser, J. A. et al. An extended hardness limit in bulk nanoceramics. Acta Mater. 69, 9-16 (2014).

5. Schiøtz, J. \& Jacobsen, K. W. A maximum in the strength of nanocrystalline copper. Science 301, 1357-1359 (2003).

6. Rupert, T. J., Gianola, D. S., Gan, Y. \& Hemker, K. J. Experimental observations of stress-driven grain boundary migration. Science 326, 1686-1690 (2009).

7. Lu, K., Lu, L. \& Suresh, S. Strengthening materials by engineering coherent internal boundaries at the nanoscale. Science 324, 349-352 (2009).

8. Reddy, K. M. et al. Enhanced mechanical properties of nanocrystalline boron carbide by nanoporosity and interface phases. Nat. Commun 3, 1052 (2012).

9. Lu, L., Chen, X., Huang, X. \& Lu, K. Revealing the maximum strength in nanotwinned copper. Science 323, 607-610 (2009).

10. Wang, J.-W. et al. Near-ideal theoretical strength in gold nanowires containing angstrom scale twins. Nat. Commun 4, 1742 (2013).

11. Tian, Y.-J. et al. Ultrahard nanotwinned cubic boron nitride. Nature 493, 385-388 (2013).

12. Huang, Q. et al. Nanotwinned diamond with unprecedented hardness and stability. Nature 510, 250-253 (2014).

13. Hubert, H. et al. Icosahedral packing of B12 icosahedra in boron suboxide (B6O). Nature 391, 376-378 (1998).

14. Chen, C. et al. $\mathrm{B}_{6} \mathrm{O}$-based composite to rival polycrystalline cubic boron nitride. Adv. Mater. 19, 4288-4291 (2007).

15. Lu, Y. P. \& He, D.-W. Structure and elastic properties of boron suboxide at 240 GPa. J. Appl. Phys 105, 083540 (2009).

16. Varley, J. B., Lordi, V., Miglio, A. \& Hautier, G. Electronic structure and defect properties of $\mathrm{B} 6 \mathrm{O}$ from hybrid functional and many-body perturbation theory calculations: A possible ambipolar transparent conductor. Phys. Rev. B 90, 045205 (2014). 
17. Reddy, K. M. et al. Shear amorphization of boron suboxide. Scr. Mater 76, 9-12 (2014).

18. Reddy, K. M., Liu, P., Hirata, A., Fujita, T. \& Chen, M.-W. Atomic structure of amorphous shear bands in boron carbide. Nat. Commun 4, 2483 (2013).

19. Domnich, V., Reynaud, S., Haber, R. A. \& Chhowalla, M. Boron carbide: structure, properties, and stability under stress. J. Am. Ceram. Soc. 94, 3605-3628 (2011)

20. An, Q., Goddard, III. W. A. \& Cheng, T. Atomistic explanation of shearinduced amorphous band formation in boron carbide. Phys. Rev. Lett. 113, 095501 (2014).

21. An, Q. \& Goddard, III W. A. Atomistic origin of brittle failure of boron carbide from large-scale reactive dynamics simulations: suggestions toward improved ductility. Phys. Rev. Lett. 115, 105501 (2015).

22. An, Q. \& Goddard, III W. A. Boron suboxide and boron subphosphide crystals: hard ceramics that shear without brittle failure. Chem. Mater. 27, 2855-2860 (2015).

23. Wade, K. The structural significance of the number of skeletal bonding electron-pairs in carboranes, the higher boranes and borane anions, and various transition-metal carbonyl cluster compounds. J. Chem. Soc. D 15, 792-793 (1971).

24. Sano, T. \& Randow, C. L, The effect of twins on the mechanical behavior of boron carbide. Metall. Mater. Trans. A 42, 570-574 (2011).

25. Oleinik, G. S. \& Ostapchuk, T. V. Effect of pores on the cleavage of twinned boron carbide crystals. Powder Metall. Met. Ceram 34, 500-504 (1995).

26. Ruh, R., Kearns, M., Zangvil, A. \& Xu, Y. Phase and property studies of boron carbide-boron nitride composites. J. Am. Ceram. Soc. 75, 864-872 (1992).

27. Rosengaard, N. M. \& Skriver, H. L. Calculated stacking-fault energies of elemental metals. Phys. Rev. B 47, 12865-12873 (1993).

28. Chisholm, J. A. \& Bristowe, P. D. A first principles investigation of stacking fault energies and bonding in wurtzite materials. J. Phys.: Condens. Matter 11, 5057-5063 (1999).

29. Becke, A. D. \& Edgecombe, K. E. A simple measure of electron localization in atomic and molecular systems. J. Chem. Phys. 92, 5397-5403 (1990).

30. Fujita, T. et al. Assymetric twins in rhombohedral boron carbide. Appl. Phys. Lett. 104, 021907 (2014).

31. Kresse, G. \& Hafner, J. Ab initio molecular dynamics for liquid metals. Phys. Rev. B. 47, 558-561 (1993).

32. Kresse, G. \& Furthmuller, J. Efficiency of ab-initio total energy calculations for metals and semiconductors using a plane-wave basis set. J. Comput. Mater. Sci. 6, 15-50 (1996).

33. Kresse, G. \& Furthmüller,, J. Efficient iterative schemes for ab initio totalenergy calculations using a plane-wave basis set. Phys. Rev. B 16, 11169-11186 (1996).

34. Kresse, G. \& Joubert, D. From ultrasoft pseudopotentials to the projector augmented-wave method. Phys. Rev. B 59, 1758-1775 (1999).
35. Roundy, D., Krenn, C. R., Cohen, M. L. \& Morris, Jr. J. W. Ideal shear strengths of fcc aluminum and copper. Phys. Rev. Lett 82, 2713-2716 (1999).

36. Li, B., Sun, H. \& Chen, C.-F. Large indentation strain-stiffening in nanotwinned cubic boron nitride. Nat. Commun 5, 4965 (2014).

37. Akashi, T., Itoh, T., Gunjishima, I., Masumoto, H. \& Goto, T. Thermoelectric properties of hot-pressed boron suboxide $\left(\mathrm{B}_{6} \mathrm{O}\right)$. Mater. Trans. 43, 1719-1723 (2002).

38. Guo, J.-J. et al. Nanoindentation characterization of deformation and failure of aluminum oxynitride. Acta Mater. 59, 1671-1679 (2011).

\section{Acknowledgements}

This work was supported by the Defense Advanced Research Projects Agency (W31P4Q13-1-0010, program managers, Judah Goldwasser and John Paschkewitz), by the Army Research Laboratory under Cooperative Agreement Number W911NF-12-2-0022 and by the National Science Foundation (DMR-1436985, program manager, John Schlueter). The experimental work was carried out at WPI-AIMR, Tohoku University, Japan, with research supported by the 'World Premier International (WPI) Center Initiative Atoms, Molecules and Materials', MEXT, Japan. We thank Professor Goto of IMR at the Tohoku University for providing as-synthesized $\mathrm{B}_{6} \mathrm{O}$ sample for experimental observations. We thank Dr Kelvin Xie in the Johns Hopkins University for useful discussion.

\section{Author contributions}

Q.A., K.M.R. and W.A.G. wrote the manuscript. Q.A. and J.Q. performed the QM simulations and K.M.R. conducted the TEM characterization and performed image simulation. Q.A., K.M.R., K.H., M.-W.C. and W.A.G. analysed the data and discussed the results. All authors commented on the manuscript.

\section{Additional information}

Supplementary Information accompanies this paper at http://www.nature.com/ naturecommunications

Competing financial interests: The authors declare no competing financial interests.

Reprints and permission information is available online at http://npg.nature.com/ reprintsandpermissions/

How to cite this article: An, Q. et al. Nucleation of amorphous shear bands at nanotwins in boron suboxide. Nat. Commun. 7:11001 doi: 10.1038/ncomms11001 (2016).

(c) (i) This work is licensed under a Creative Commons Attribution 4.0 International License. The images or other third party material in this article are included in the article's Creative Commons license, unless indicated otherwise in the credit line; if the material is not included under the Creative Commons license, users will need to obtain permission from the license holder to reproduce the material. To view a copy of this license, visit http://creativecommons.org/licenses/by/4.0/ 